\title{
Printed Materials Available from the NALld Publications Center
}

YOU must be an NALLD member to use this service. In many cases the reprint itself is free; however, you must provide a self-addressed, $9 \times 12$ inch manila envelope that is stamped with the proper return postage. The order form lists the weight of each item; you must total up the weight of all items ordered and compute your postage after deciding whether you want the information to be sent by First Class or Third Class mail. Requests for materials will be honored only when made on the order form and accompanied by the properly stamped selfaddressed manila envelope.

At present we cannot distribute items in quantity; however, you have our permission to reproduce or copy any material received from the Publications Center provided you identify the source by the line:

\section{-Reprinted by permission of the National Association of Language Laboratory Directors}

NALLD members are encouraged to offer items for possible inclusion in the Publications Center. Please address Mr. Charles P. Richardson, Director, NALLD Publications Center, Ellis Hall, Ohio University, Athens, Ohio 45723 . We would especially like routine work forms which schools actually use in their operations. Naturally, you must agree that others can reproduce any material provided to our Publications Center.

Members are encouraged to offer suggestions on other categories which we should add to our lists.

Item

\section{Description}

Number

1.01.00 NALLD OFFICIAL BUSINESS - Reports to Members.

.01 Copy of NALLD Constitution, revised in May, 1967.

.02 Minutes of the Second Final Organizational Meeting of NALLD (28 April 1965, Milwauke,, Wisconsin at DAVI Convention).

.03 Memo of 2 August 1965, giving further information of NALLD $\&$ its plans.

.04 Minutes of NALLD Midwest Regional Meeting (December 1965, Chicago, Illinois at MLA Convention).

.05 Minutes of First Annual Business Meeting (April 1966, San Diego, Calif., at DAVI Convention).

.06 Minutes of Southern Regional Meeting (1966, Atlanta, Georgia at Southern Conference on Language Teaching). 
1.01.07 Minutes of Eastern Regional Meeting (December 1966, New York, N. Y., at MLA Convention). Also, Membership Report from the Secretary-Treasurer for December 1966.

.08 Minutes of Second Annual Business Meeting (April 1967, Atlantic City, N. J., at DAVI Convention).

1.02.00 NALLD SELECTED SPEECHES \& POLICY STATEMENTS.

.01 "Should There Be a Language Laboratory Director at the Secondary Level?" Speech by Dr. J. Michael Moore, San Dicgo City Schools. (NALLD First Annual Business Meeting, April 1966, San Diego).

.02 Policy Statement To Textbook Publishers: "Resolution Urging Publishers To Provide Suitable \& Comprehensive Guides \& Scripts for Tapes."

.03 .00 NALLD NEWSLETTERS - BACK ISSUES.

.01 Volume I, Number 1 (February, 1966)

.02 Volume I, Number 2 (September, 1966)

.03 Volume I, Number 3 (February, 1967)

.04 Volume II, Number 1 (December, 1967)

2.00.00 SAMPLE COPIES OF WORKING SYSTEMS: DESCRIPTIONS, INSTRUCTIONS, MANUALS.

.01 .00 Job Description: Language Laboratory Director

.01 (may we have your school's, please?)

.02 .00 Job Description: Chief Technician (Technical Supervisor)

.01 University of Wisconsin - Madison (Civil Service, Full time)

.02 University of Hawaii

.03 .00 Job Description: Laboratory Secretary

.01 (may we have your school's description, please?)

.04 .00 Job Description: Tape Processing Supervisor

.05 .00 Job Description: (Student) Open-Hour Proctor

.06 .00 Job Description: (Student) Recording Session Technician

.07 .00 Job Description: (Student) Minor Repair \& Maintenance Technician

.08.00 Job Description: (Student) Tape Library Processing

.01 University of Wisconsin - Madison (Five separate booklets covering different phases: Stamping, Duplicating, Banding \& Filling-In Worksheets, Typing Up Final TICC Guide, \& Final Check.)

.09.00 Tape Locator Catalog - Sample Copy

.01 University of Wisconsin - Madison

.02 A sample copy from your school? 
2.10.00 Student Orientation Guide To Language Laboratories

.01 University of Wisconsin - Madison

.02 A sample copy from your school?

.11 .00 Faculty Semester Information Packet

.01 University of Wisconsin - Madison

.12 .00 What else do you suggest we add here ???

3.00.00 SAMPLE COPIES OF BLANK FORMS USED

.01 .00 In Language Lab Office \& Lab Administration

.01 University of Wisconsin - Madison

.02 Ohio University - Athens, Ohio (Charles Richardson)

.03 Wheaton College - Norton, Massachusetts

(Mrs. Jean S. Conkey)

.04 State University College - New Paltz, New York (Henry Urbanski)

.02 .00 In the Language Labs

.01 University of Wisconsin - Madison

.02 Ohio University - Athens, Ohio

.03 A sample from your school?

.03 .00 Used by Lab Technicians for Repair, Maintenance, \& Preventive Maintenance.

.01 University of Wisconsin - Madison

.04 .00 Used to Process Tapes into Language Laboratory Regular Tape Library. (Including Recording Sessions, Duplication, Cataloging, \& Indexing.)

.01 University of Wisconsin - Madison

.05 .00 Dial Access Program Library Operation

.01 University of Wisconsin - Madison (preliminary forms)

.02 Sample material from your school?

4.00.00 TECHNICAL DATA PREPARED BY SCHOOLS FOR PROCURING OR PLANNING FACILITIES

.01 .00 Functional \& Technical Specifications for Regular Booth Language Labs.

.01 (category open - contributions desired)

.02 .00 Functional \& Technical Specifications for Remote Record Lab

.01 (category open - contributions desired)

.03.00 Functional \& Technical Specifications for Dial-Access Labs

.01 (category open - contributions desired)

.04 .00 Functional \& Technical Specifications for Overhead Labs

.01

.05 .00 (category open - contributions desired)

.01 "Reasons for Using 2 Track Rather than 4 Track Tapes as

Program Sources in Dial-Access Retrieval Systems" T.R.

Goldsworthy, University of Wisconsin - Madison 
4.05.02 "Cost Analysis of Expanding Automatic Dial-Access Switching Systems," Workpaper T.R. Goldsworthy, University of Wisconsin - Madison

5.00.00 PEDAGOGICAL \& OPERATIONAL ORIENTATION OF SCHOOL STAFF WHEN PLANNING FACILITIES

.01 .00 Background Information Papers to Faculty

.01 University of Wisconsin - Madison (Several papers concerning new building facilities with dial-access $\&$ remote record.)

.02 Sample material from your school?

6.00.00 INFORMATION PAPERS, MEMORANDA

.01 .00 (category open - contributions desired)

7.00.00 LANGUAGE LAB TECHNIQUES - MATERIALS FOR COURSES \& WORKSHOPS (COURSE DESCRIPTIONS, OUTLINES, BIBLIOGRAPHIES, SAMPLE LESSONS)

.01 .00 (category open - contributions desired)

8.00.00 TAPE INDEX CORRELATION CODE (TICC) GUIDES These guides were prepared to give close correlation between sets of tapes used at one school with their textbooks, by relating tape bands \& reels with textbook pages, chapters, \& grammatical topics. All these guides are predicated upon the fact that the lab tapes are recorded in one direction only (on one track only) so that they can be subdivided. Those who would care to prepare similar guides for other textbooks are invited to do so.

.01 .00 French TICC Guides - Beginning Level

.01 Harris \& Leveque, Basic Conversational French, Revised 3rd Edition, Conversations. (Holt, 1962) FR 1.001.43

.02 Harris \& Leveque, Basic Conversational French, Revised 3 rd Edition, Grammar Units. (Holt, 1962) FR 1.002.25

.03 Heise \& Muller, A Conversational Introduction to French. (Dodd, Mead \& Co., 1963) FR1.003.15

.04 Palmeri, Conversational \& Cultural French.

(Appleton-Century-Crof ts, 1966) FR1.005.04

.02 .00 French TICC Guides-Intermediate Level

.01 Bottke, Brief Oral Frencb Review (Appleton, 1964)

FR2.001.08

.02 Harris, Intermediate Conversational French, Revised (Holt, 1966) FR2.003.

.03 Mondelli, French Conversational Review Grammar, 2nd Edition (American Book Co., 1961) FR2.005.10

.03.00 French TICC Guides - Advanced Level

.01 Leon, Excrcises Systematiques de Prononciation Francaise. (Paris: Librairies Hachette et Larousse, 1964) FR3.001.06 
Valdman, $A$ Drillbook of French Promunciation. (Harper \& Row, 1964) FR3.004.20

.04 .00 German TICC Guides - Beginning Level

.01 Mathieu, Quick Cbange Audio-Drills in Fundamental German. (Regents Publishing Co., 1961) GR1.001.72

.02 Scherer, Contemporary German. (McGraw-Hill, 1966) GR1.002.45

.05 .00 German TICC Guides - Intermediate Level

.01 Mathieu, Expanded Quick-Change Audio-Drills in German. (Regents Publishing Co., 1963) GR2.001.82

.02 Rehder, et al., Lesen und Denken. (Holt, 1964) GR2.002.18 Griesbach, Deutsche Spracblebre fuer Auslaender. Munich: Max Hueber, 1964) GR2.004.04 German TICC Guides - Advanced Level (not completed yet)

Italian TICC Guides - Beginning Level Hall, Basic Conversational Italian. (Holt, 1963) IT 1.001.24

Italian TICC Guides - Intermediate Level (not completed yer) Italian TICC Guides - Advanced Level (not completed yet)

.10 .00 Spanish TICC Guides - Beginning Level

.01 MLA, Modern Spanish, 1st Edition. (Harcourt, Brace, 1960) SP1.001.45 MLA, Modern Spanish, 1st Edition. (Dialogues) (Harcourt, Brace, 1960) SP1.002.04

.03 Maestas, Teacher's Manual E Guide - Spanish Linguatapes Series M. (St. Louis: Edu-Tek, Inc., 1962) SP1.003.08

.04 Rogers, Spanish for the First Year. (MacMillian Co., 1964) SP1.005.19

.05 Leslie, Spanish for Conversation. (Ginn \& Co., 1959) SP1.006.23

.06 Florit, Escuche y Acierte. McGraw-Hill, 1965) SP1.009.06

.11 .00 Spanish TICC Guides - Intermediate Level

.01 O'Connor, et al., Oral Drill in Spanish, 2nd Edition. (Houghton-Mifflin Co., 1963) SP2.001.22

.02 Wise, Teacher's Manual and Guide for Linguatapes Pattern Drills Series $A$ (St. Louis, Edu-Tek, 1961) SP2.002.20

.03 Urgarte, Gramatica Espanola de Repaso, (Odyssey Press, 1958) SP2.003.08

.04 Yates, Imaginacion y Fantasia.

(Holt, 1960) SP2.004.22 


\section{NALLD PUBLICATIONS CENTER ORDER FORM}

To obtain materials send this order form properly completed along with a $9 \times 12$ inch self-addressed manila envelope properly stamped with the correct postage to:

\section{Mr. Charles Richardson, Director \\ NALLD Publications Center \\ Ellis Hall \\ Ohio University \\ Athens, Ohio 45723}

Please send the items checked below to the following address:

Name Title

Address

City

State

Zip Code

Summary of order: Total number of items desired

Total weight in ounces

Multiply weight times postage for the class of mail you desire to compute stamps necessary.

Approx.

$\begin{array}{ll} & \text { Item Weight In } \\ \text { Quant. Number OUNCISS }\end{array}$

Approx.

Approx.

Item Weight In

Item Weight In

$\begin{array}{ll}\text { ( ) } & 1.01 .01 \\ \text { ( ) } & 1.01 .02 \\ \text { ( ) } & 1.01 .03 \\ \text { ( ) } & 1.01 .04 \\ \text { ( ) } & 1.01 .05 \\ \text { ( ) } & 1.01 .06 \\ \text { ( ) } & 1.01 .07 \\ \text { ( ) } & 1.01 .08 \\ \text { ( ) } & 1.02 .01 \\ \text { ( ) } & 1.02 .02 \\ \text { ( ) } & 1.03 .01 \\ \text { ( ) } & 1.03 .02 \\ \text { ( ) } & 1.03 .03 \\ \text { ( ) } & 1.03 .04 \\ \text { ( ) } & 2.02 .01 \\ \text { ( ) } & 2.02 .02 \\ \text { ( ) } & 2.04 .01 \\ \text { ( ) } & 2.05 .01 \\ \text { ( ) } & 2.06 .01 \\ \text { ( ) } & 2.07 .01\end{array}$

0.50

Quant. Number OUNCES

Quant. Number OUNCES

$\begin{array}{ll}\text { ( ) } & 2.08 .01 \\ \text { ( ) } & 2.09 .01 \\ \text { ( ) } & 2.10 .01 \\ \text { ( ) } & 2.11 .01 \\ \text { ( ) } & 3.01 .01 \\ \text { ( ) } & 3.01 .02 \\ \text { ( ) } & 3.01 .03 \\ \text { ( ) } & 3.01 .04 \\ \text { ( ) } & 3.02 .01 \\ \text { ( ) } & 3.02 .02 \\ \text { ( ) } & 3.03 .01 \\ \text { ( ) } & 3.04 .01 \\ \text { ( ) } & 3.05 .01 \\ \text { ( ) } & 4.05 .01 \\ \text { ( ) } & 4.05 .02 \\ \text { ( ) } & 5.01 .01 \\ \text { ( ) } & 8.01 .01 \\ \text { ( ) } & 8.01 .02 \\ \text { ( ) } & 8.01 .03 \\ \text { ( ) } & 8.01 .04\end{array}$

7.50
2.75
2.75
1.50
3.50
0.50
0.50
0.50
2.00
0.75
2.00
2.00
0.25
0.50
1.00
1.50
3.00
1.75
1.00
0.75

( ) 8.02 .01

0.75

0.25

0.25

0.25

0.25

0.25

0.25

0.25

2.00

0.25

1.00

1.00

1.50

1.50

1.50

0.50

2.00

2.00

2.00

2.00

$\begin{array}{lll}\text { ( ) } 8.02 .01 & 0.75 \\ \text { ( ) } 8.02 .02 & 2.00 \\ \text { ( ) } 8.02 .03 & 0.75 \\ \text { ( ) } 8.03 .01 & 1.00 \\ \text { ( ) } 8.03 .02 & 1.50 \\ \text { ( ) } 8.04 .01 & 0.75 \\ \text { ( ) } 8.05 .01 & 2.50 \\ \text { ( ) } 8.05 .02 & 0.50 \\ \text { ( ) } 8.05 .03 & 0.75 \\ \text { ( ) } 8.07 .01 & 1.50 \\ \text { ( ) } 8.10 .01 & 2.50 \\ \text { ( ) } 8.10 .02 & 0.50 \\ \text { ( ) } 8.10 .03 & 1.75 \\ \text { ( ) } 8.10 .04 & 0.75 \\ \text { ( ) } 8.10 .05 & 0.75 \\ \text { ( ) } 8.10 .06 & 1.50 \\ \text { ( ) } 8.11 .01 & 2.50 \\ \text { ( ) } 8.11 .02 & 2.00 \\ \text { ( ) } 8.11 .03 & 0.75 \\ \text { ( ) } 8.11 .04 & 1.00\end{array}$

